\title{
Internal Waves Under the Arctic Pack Ice During the Arctic Internal Wave Experiment: The Coherence Structure
}

\author{
MurRay D. Levine \\ College of Oceanography, Oregon State University, Corvallis
}

\begin{abstract}
The spectral composition of internal gravity waves under the Arctic pack ice during the Arctic Internal Wave Experiment (AIWEX) was found to be strikingly different from observations at lower latitudes. Time series of vertical displacement were inferred from horizontal and vertical arrays of temperature and conductivity sensors. Frequency spectra indicate a whiter spectrum (spectral slope near -1 ) and a less energetic wave field (by a factor of 0.02 ) than observations at lower latitude. The analysis of vertical and horizontal coherences revealed a horizontally isotropic wave field that is consistent with assumptions of a random field of linear internal waves. The wavenumber bandwidth of the wave field is about a factor of 10 wider than found at lower latitude.
\end{abstract}

\section{INTRODUCTION}

Internal gravity waves are ubiquitous in the world's oceans. Kinematically, they are responsible for much of the temporal variability of velocity and density at frequencies between $f$ and $N$. Dynamically, internal waves provide the lirk that transfers energy and momentum from large-scale, nearly two-dimensional circulation to small-scale, threedimensional fluctuations. Complex nonlinear interactions among the waves undoubtedly are important in this transfer [Müller et al., 1986]. Despite their role in the overall energy and momentum balance of the ocean, the identification of the specific processes that generate and dissipate internal waves remains elusive. Theoretical studies indicate that many mechanisms are potentially important; yet it has been difficult to determine experimentally which of these is most significant to the ocean.

While it is difficult to uncover the sources and sinks of the waves, observations can tell us the frequency-wavenumber composition of the wave field. Internal waves are observed to be remarkably steady and homogeneous. Nearly two decades ago, Garrett and Munk [1972] (hereinafter called GM) exploited this observation and constructed a spectral model describing the steady-state internal wave field. The main assumption of the GM model is that the observed confused sea of velocity fluctuations can be represented as a random sum of linear internal waves. The model incorporates the kinematic constraints of internal waves, namely the dispersion relation, and requires the wave field to be in equilibrium, that is, statistically steady in time and homogeneous in space. The essence of the model is the specification of the frequency-wavenumber distribution of the internal wave energy. Once determined, this energy distribution function can be used to evaluate any type of measurable statistical quantity. In this way the GM model provides a framework for comparing diverse observations from the same experiment as well as comparing similar types of measurements from different experiments.

The quantities that have been used most extensively for comparing internal waves from one experiment to another are the variances of horizontal velocity and vertical displace-

Copyright 1990 by the American Geophysical Union.

Paper number 89JC03683.

0148-0227/90/89JC-03683\$05.00 ment in the internal wave frequency band. Wunsch [1976] and Wunsch and Webb [1979] tabulated the energy in the wave field from diverse oceanic experiments and found the estimates to be within a factor of about 3 , except for observations near abrupt topography. It was suggested by Wunsch [1975] that clues to the identity of the major sources and sinks in the internal wave energy balance might be gained by searching for regions where the internal wave field is most different from the canonical GM model. Levine et al. [1985] updated this internal wave climatology and found evidence that the internal wave energy levels in the Arctic Ocean might be lower than in ice-free oceans. With the prospect of observing an internal wave field that was unlike most other previous observations, the Arctic Internal Wave Experiment (AIWEX) was organized.

The experiment took place over deep water in the Beaufort Sea at an ice camp about $350 \mathrm{~km}$ north of Prudhoe Bay, Alaska, for nearly 2 months from March to May 1985 (Figure 1). The internal wave field observed during AIWEX was indeed significantly less energetic than lower-latitude observations, by a factor of 0.03 to 0.07 [Levine et al., 1987]. In addition, the shape of the frequency spectrum was much different than any previous observation. The spectral slope was nearly -1 rather than the -2 of the GM model, which was based primarily on mid-latitude observations. Spectral slopes differing from the GM value of -2 have been reported [e.g., Pinkel, 1983; Levine et al., 1983a], but none whiter than about -1.5 .

While interesting, the frequency autospectra do not provide insight into the wavenumber composition of the wave field. The structure of the coherences between spatially separated locations provides information about the frequency-wavenumber distribution of the wave field. Under the assumption of the wave field as a random sea of waves, the coherence at a given frequency is reduced from its maximum value of 1 by the presence of waves of more than one wavelength - the wider the wavenumber bandwidth of the waves, the lower the coherence [Munk and Phillips, 1968].

The purpose of this paper is to present the results of an analysis of the average coherence structure of the internal wave field based on vertical displacement time series measured during AIWEX. Unlike frequency spectra, the climatology of the coherence structure of the wave field is not well established. However, the average vertical and horizontal 


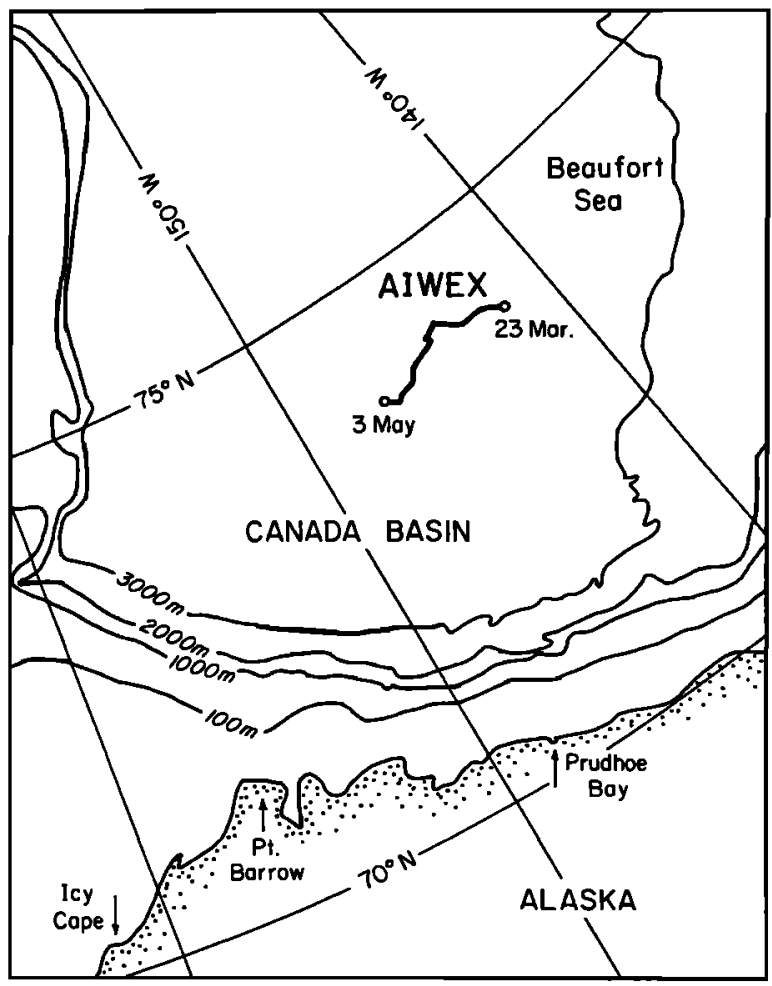

Fig. 1. Map indicating the position of the ice camp in the Beaufort Sea of the Arctic Ocean as it drifted to the west during AIWEX.

coherences at AIWEX were found to be strikingly low compared to previous open ocean observations. Despite the low level, the structure of the observed coherences was consistent with a random field of horizontally isotropic, linear internal waves. The experiment and observations are described in section 2 . A discussion of modeling the internal wave field as a random process is presented in section 3 along with the theoretical considerations needed for extracting the frequency-wavenumber energy distribution from horizontal and vertical coherences. In section 4 the theory is applied to the data and the results compared with previous studies. A conclusion and summary of the analysis and observations are given in section 5 .

\section{EXPERIMENTAL Description}

A vertical and horizontal array of temperature and conductivity sensors was used to measure time series of vertical displacement due to internal waves (Figure 2). The horizontal array consisted of sensors at six satellite locations, nominally at a depth of $250 \mathrm{~m}$, forming two equilateral triangles. The sides of the outer triangle were about $1 \mathrm{~km}$; the sides of the inner triangle were near $250 \mathrm{~m}$. The deployment of this unique, small-scale horizontal array over deep water was feasible because of the stable platform provided by the Arctic ice pack; the construction of a similar array in a temperate ocean would be extremely difficult. The vertical array consisted of sensors at seven depths on a single mooring in the center of the triangles. The temperature and conductivity sensors were manufactured by Sea-Bird Electronics (Oceanographic Thermometer SBE-3; Conductivity Meter SBE-4). The sensors' output is a frequency that is proportional to the temperature or conductivity. Individual shielded, three-conductor wire cables from each sensor carried the signal to a hut located at the central mooring. Power to the sensors was also provided on the same cable. In the hut all the sensor frequencies were measured simultaneously with a 20-channel Sea-Bird Counter (SBE 11/20), and then averaged and recorded every minute on floppy disk using a PDP 11/23. Pressure sensors manufactured by Paroscientific (Digiquartz model 8600) were attached to the bottom of $\mathrm{I} 3$ and the central mooring to monitor tilt.

The array was installed at $74^{\circ} \mathrm{N}, 143^{\circ} \mathrm{W}$ and drifted with the ice nearly $120 \mathrm{~km}$ to the west during the experiment (Figure 1). The moorings consisted of $\frac{1}{4}$-inch synthetic line with the wire cables and sensors attached by black tape and nylon ties. The moorings were deployed through holes drilled in the 3-m thick ice with the top secured firmly to the ice. To keep the mooring taut, masses were attached at the bottom- $45 \mathrm{~kg}$ on the satellite moorings and $90 \mathrm{~kg}$ on the
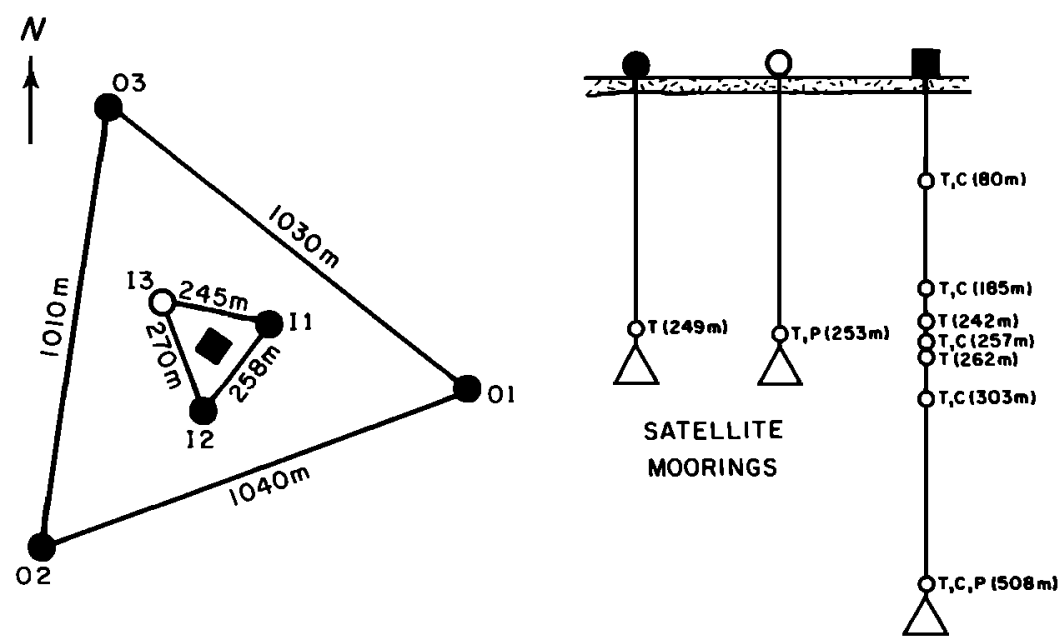

CENTRAL

MOORING

Fig. 2. Schematic of the horizontal and vertical array of temperature (T), conductivity (C), and pressure (P) sensors deployed during AIWEX. 


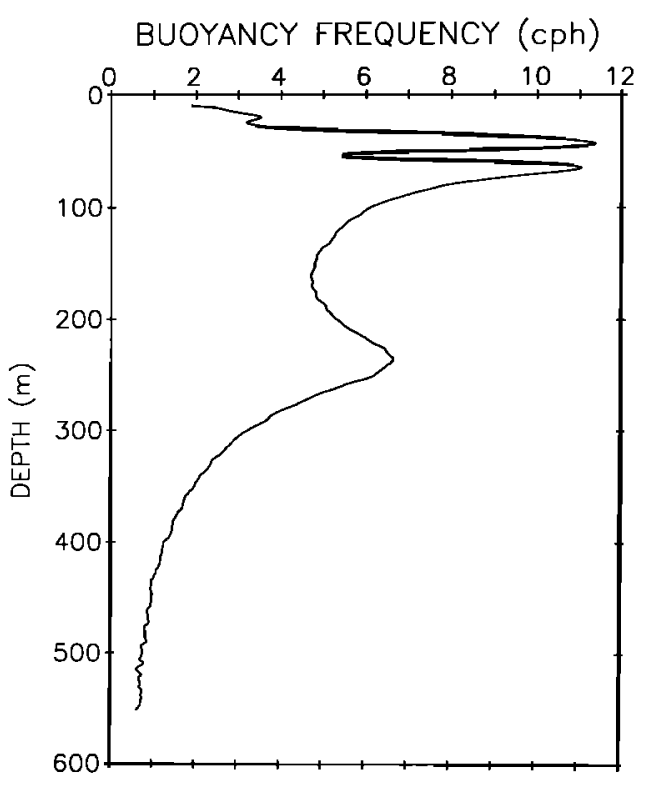

Fig. 3. Average buoyancy frequency profile for the period March 21 to April 11 (J. Morison, personal communication, 1989).

central. The data were recorded for about 5 weeks from March 18 until April 29.

The response time of the temperature sensors is $200 \mathrm{~ms}$, and there was no aliasing in the one minute samples as the signal was averaged over the entire minute. However, the response time of the conductivity cell is not easily determined as it is controlled by the flushing rate of the water through a tube $0.2 \mathrm{~m}$ long and $4-7 \mathrm{~mm}$ in diameter. The difference in response times of the sensors can lead to problems in estimating salinity. A simple model was made of the flushing of the conductivity cell, and it was determined that the best estimate of salinity was obtained using the temperature record after smoothing with a single-pole filter with a 4 minute time constant (for details, see Levine et al. [1986a]). The filtering was done in order to approximate the temperature of the water inside the conductivity cell. Although this is only an approximate method, it is entirely adequate to resolve frequencies in the internal wave band.

To investigate the internal wave field, the temperature and salinity data were converted to vertical displacement using daily-averaged profiles made with the Arctic Profiling System (APS) by J. Morison. The daily-averaged profiles were calculated typically from eight casts; vertical gradients of temperature and salinity were then estimated from a $10 \mathrm{~m}$ section of data centered at the depth of each sensor. Around depths of 250 and $300 \mathrm{~m}$ both the temperature and salinity gradients were stable enough to allow conversion to vertical displacements; at 80 and $185 \mathrm{~m}$ only salinity gradients were stable. The temperature and salinity gradients at $500 \mathrm{~m}$ were too small to permit a reliable determination of vertical displacement, and hence these data are not used in the analysis. The time variability of the vertical gradient was generally small except during the period when an eddy was present (April 23-29). The analysis presented here does not include the eddy period; it is left for another study.

An average buoyancy frequency profile was also calculated from the APS data (Figure 3). This profile was calculated from the density profile averaged between March 21 and April 11. The highest values of $N$ are near $12 \mathrm{cph}$ in the upper $100 \mathrm{~m}$; below $250 \mathrm{~m}$ the buoyancy frequency decays nearly exponentially.

\section{Theoretical Considerations}

By assuming the wave field consists of a sum of free, random waves, Garrett and Munk [1972] were able to construct a consistent, statistical model that is successful in describing many diverse observations of the internal wave field. The main tenets of the GM model will be used here to model the internal waves during AIWEX. However, the formulation is kept as general as possible in order to highlight the unique features of the AIWEX data.

Let the vertical displacement of the internal wave field at frequency $\omega$ be represented as a random process $\zeta(x, \omega)$, and the spectral representation of the process be given by

$$
\begin{array}{r}
\zeta(x, \omega)=\sum_{s} \int_{-\infty}^{\infty} \int_{-\infty}^{\infty} A(k, l, \omega, s) \exp [i(k x+l y)] \nu(z) \\
\cdot \exp [i s \phi(z, k, l, \omega)] d k d l
\end{array}
$$

where $A$ is the spectral measure of the process and $(k, l)$ are the horizontal wavenumbers in the $(x, y)$ directions respectively, and $s$ is an index that is either + or - . The depth dependence of the wave is expressed by using the WKBJ approximation, which requires the vertical wavelength of the wave to be small relative to the vertical variation in $N(z)$ [e.g., Gill, 1982]. It is also assumed, as in GM, that the frequency $\omega$ is much less than the local value of the buoyancy frequency $N(z)$. The depth dependence of the amplitude of vertical displacement can then be written as a non-dimensional function

$$
\nu(z)=\left[\frac{\tilde{N}^{2}-\omega^{2}}{N^{2}(z)-\omega^{2}}\right]^{1 / 4}=\left[\frac{\tilde{N}}{N(z)}\right]^{1 / 2}
$$

where $\tilde{N}$ is a reference buoyancy frequency. The depth dependence of phase is given by:

$$
\phi(z, k, l, \omega)=\left[\frac{k^{2}+l^{2}}{\omega^{2}-f^{2}}\right]^{1 / 2} \int_{z_{*}}^{z}\left[N^{2}(z)-\omega^{2}\right]^{1 / 2} d z
$$

where $z_{*}$ is the turning depth, defined by $\omega=N\left(z_{*}\right)$. The sign $s$ accounts for both upward and downward propagating waves.

The frequency cross-spectrum of $\zeta$ at two locations in space then becomes

$$
\begin{aligned}
& S_{c}\left(\Delta x, \Delta y, z_{1}, z_{2}, \omega\right) \\
&=\left\langle\zeta\left(x, y, z_{1}, \omega\right) \zeta^{*}\left(x+\Delta x, y+\Delta y, z_{2}, \omega\right)\right\rangle \\
&= \sum_{s} \sum_{s^{\prime}} \int_{-\infty}^{\infty} \int_{-\infty}^{\infty} \int_{-\infty}^{\infty} \int_{-\infty}^{\infty}\left\langle A(k, l, \omega, s) A^{*}\right. \\
&\left.\cdot\left(k^{\prime}, l^{\prime}, \omega, s^{\prime}\right)\right\rangle \nu\left(z_{1}\right) \nu\left(z_{2}\right) \\
& \cdot \exp \left\{i \left[\left(k-k^{\prime}\right) x+\left(l-l^{\prime}\right) y\right.\right. \\
&\left.\left.+s \phi\left(z_{1}, k, l, \omega\right)-s^{\prime} \phi\left(z_{2}, k^{\prime}, l^{\prime}, \omega\right)\right]\right\} \\
& \cdot \exp \left[-i\left(k^{\prime} \Delta x+l^{\prime} \Delta y\right)\right] d k d k^{\prime} d l d l^{\prime}
\end{aligned}
$$


where $\langle\cdot\rangle$ indicates expected value or ensemble average. The subscript $c$ denotes that the cross-spectrum is defined using a Cartesian coordinate system. To insure that the random process $\zeta$ is homogeneous in $x$ and $y$, and upward- and downward-propagating waves are uncorrelated, it is required that

$$
\begin{aligned}
\left\langle A(k, l, \omega, s) A^{*}\right. & \left.\left(k^{\prime}, l^{\prime}, \omega, s^{\prime}\right)\right\rangle \\
& =E_{c}(k, l, \omega, s) \delta\left(k-k^{\prime}, l-l^{\prime}, s-s^{\prime}\right)
\end{aligned}
$$

[e.g., Müller et al., 1978], where $E_{c}$ is the spectral energy distribution function at depth $z$ where $N(z)=\tilde{N}$. The cross-spectrum (4) then becomes

$$
\begin{aligned}
S_{c}\left(\Delta x, \Delta y, z_{1}, z_{2}, \omega\right)= & \sum_{s} \int_{-\infty}^{\infty} \int_{-\infty}^{\infty} E_{c}(k, l, \omega, s) \\
& \cdot \exp [-i(k \Delta x+l \Delta y)] \nu\left(z_{1}\right) \nu\left(z_{2}\right) \\
& \cdot \exp \left\{i s\left[\phi\left(z_{1}\right)-\phi\left(z_{2}\right)\right]\right\} d k d l
\end{aligned}
$$

Note that for clarity the dependence of $\phi$ on $k, l$ and $\omega$ has not been explicitly indicated.

It is useful to express the horizontal position and horizontal wavenumber vectors in polar coordinates using

$$
\begin{array}{ll}
\Delta x=r \cos \psi & k=\alpha \cos \theta \\
\Delta y=r \sin \psi & l=\alpha \sin \theta
\end{array}
$$

where $\alpha=\left(k^{2}+l^{2}\right)^{1 / 2}$ is the horizontal wavenumber and $r=$ $\left(\Delta x^{2}+\Delta y^{2}\right)^{1 / 2}$ is the horizontal separation. The crossspectrum can then be written

$$
\begin{aligned}
S_{p}\left(r, \psi, z_{1}, z_{2}, \omega\right)= & \sum_{s} \int_{0}^{\infty} \int_{0}^{\pi} E_{p}(\alpha, \theta, \omega, s) \\
& \cdot \exp \{-i \alpha r[\cos (\theta-\psi)]\} \nu\left(z_{1}\right) \nu\left(z_{2}\right) \\
& \cdot \exp \left\{-i s\left[\phi\left(z_{1}\right)-\phi\left(z_{2}\right)\right]\right\} d \theta d \alpha
\end{aligned}
$$

where the subscript $p$ indicates that the cross-spectrum is defined using polar coordinates. If it is further assumed that the wave field is horizontally isotropic and vertically symmetric, then $E_{p}(\alpha, \theta, \omega, s)$ is independent of $\theta$ and $s$. The integral over $\theta$ can then be evaluated analytically [e.g., Gradshteyn and Ryzhik, 1965] and the cross-spectrum becomes

$$
\begin{aligned}
S\left(r, z_{1}, z_{2}, \omega\right)=\int_{0}^{\infty} E(\alpha, \omega) \nu\left(z_{1}\right) \nu\left(z_{2}\right) J_{0}(\alpha r) \\
\cdot \cos \left[\phi\left(z_{1}\right)-\phi\left(z_{2}\right)\right] d \alpha
\end{aligned}
$$

Note that, as expected, the cross-spectrum $S$ is not function of the orientation angle $\psi$, since the energy distribution function $E$ is not a function of $\theta$. The frequency autospectrum then becomes

$$
S(0, z, z, \omega)=\int_{0}^{\infty} E(\alpha, \omega) \nu^{2}(z) d \alpha
$$

and scales as $1 / N(z)$.
The coherence between two depths with no horizontal separation is given by

$$
\begin{aligned}
C\left(0, z_{1}, z_{2}, \omega\right) & =\frac{S\left(0, z_{1}, z_{2}, \omega\right)}{\left[S\left(0, z_{1}, z_{1}, \omega\right) S\left(0, z_{2}, z_{2}, \omega\right)\right]^{1 / 2}} \\
& =\frac{\int_{0}^{\infty} E(\alpha, \omega) \cos \left[\phi\left(z_{1}\right)-\phi\left(z_{2}\right)\right] d \alpha}{\int_{0}^{\infty} E(\alpha, \omega) d \alpha}
\end{aligned}
$$

In order to solve for $E(\alpha, \omega)$ directly from observations of vertical coherence by inverting (11), the WKBJ approximation is simplified further. Assume that $N(z)$ is nearly constant between $z_{1}$ and $z_{2}$. Then

$$
\phi\left(z_{1}\right)-\phi\left(z_{2}\right) \simeq \alpha \gamma \Delta z
$$

where

$$
\gamma=\left(\frac{\bar{N}^{2}-\omega^{2}}{\omega^{2}-f^{2}}\right)^{1 / 2}
$$

where $\bar{N}$ is a value between $N\left(z_{1}\right)$ and $N\left(z_{2}\right)$ that is nearly constant in $\omega$ for $\omega<N$. It is further assumed that $\bar{N}$ is constant for any $\Delta z$. This can be a severe restriction as it implies that $N(z)$ does not vary much for all $z$. However, in this study the observed coherences nearly reach zero before variations in $N(z)$ become significant. These assumptions permit one to take the Fourier cosine transform of $C$ (equation (11)) with respect to $\Delta z$, yielding

$$
\begin{aligned}
\bar{E}(\alpha, \omega) & =\frac{E(\alpha, \omega)}{\int_{0}^{\infty} E(\alpha, \omega) d \alpha} \\
& =\frac{2 \gamma}{\pi} \int_{0}^{\infty} C(0, \Delta z, \omega) \cos (\alpha \gamma \Delta z) d \Delta z
\end{aligned}
$$

where $\bar{E}(\alpha, \omega)$ is the normalized distribution function and the vertical coherence is assumed to be a function only of vertical separation, i.e., $C\left(0, z_{1}, z_{2}, \omega\right)=C(0, \Delta z, \omega)$ where $\Delta z=z_{2}-z_{1}$. Estimates of $E(\alpha, \omega)$ could be made without the approximation of (12), but the added complexity of the analysis is not warranted here due to the limited vertical extent of the data set.

The coherence at horizontal separation $r$ at constant depth becomes

$$
C(r, 0, \omega)=\frac{S(r, z, z, \omega)}{S(0, z, z, \omega)}=\frac{\int_{0}^{\infty} E(\alpha, \omega) J_{0}(\alpha r) d \alpha}{\int_{0}^{\infty} E(\alpha, \omega) d \alpha}
$$

Note that when using the WKBJ approximation, there is no $z$-dependence in the horizontal coherence. The inversion of the horizontal coherence to solve for $E(\alpha, \omega)$ takes the form of a Hankel transform [Bracewell, 1978], giving 


$$
\bar{E}(\alpha, \omega) \equiv \frac{E(\alpha, \omega)}{\int_{0}^{\infty} E(\alpha, \omega) d \alpha}=\alpha \int_{0}^{\infty} C(r, 0, \omega) J_{0}(\alpha r) r d r
$$

Therefore the normalized distribution $\bar{E}(\alpha, \omega)$ can be independently estimated from observations of either vertical or horizontal coherence using (13) or (15), respectively. A large number of coherence observations in the horizontal and vertical would be needed to estimate $\bar{E}$ by actually integrating (13) and (15). Since only limited observations are available from AIWEX, it is assumed that the coherence falls off exponentially with separation according to

$$
\begin{gathered}
C(0, \Delta z, \omega)=\exp [-a(\omega) \Delta z] \\
C(r, 0, \omega)=\exp [-b(\omega) r]
\end{gathered}
$$

The function $\bar{E}(\alpha, \omega)$ can then be calculated analytically from (13) and (15)

$$
\begin{aligned}
& \bar{E}_{v}(\alpha, \omega)=\frac{2 \gamma a}{\pi} \frac{1}{a^{2}+(\gamma \alpha)^{2}} \\
& \bar{E}_{h}(\alpha, \omega)=\frac{\alpha b}{\left(\alpha^{2}+b^{2}\right)^{3 / 2}}
\end{aligned}
$$

where the subscript $v$ or $h$ indicates that the quantity $E$ was calculated from vertical or horizontal coherences, respectively.

\section{OBSERVATIONS AND COMPARISON WITH THEORY}

In this section the observations are presented and compared with the theoretical ideas developed in section 3. For convenience the data are usually displayed in units of cycles per hour; the reader is alerted to account for factors of $2 \pi$ if trying to reproduce the theoretical curves presented in this section.

Spectra of vertical displacement inferred from salinity time series are shown in Figure 4. These spectra are estimated from nearly continuous data from March 21 to April 23. Each time series was divided into 12 segments of 54.5 hours, and a cosine taper was applied to the first and last $10 \%$ of each segment before the Fourier transform was taken.

To demonstrate the effect of mooring motion on the vertical displacement estimates, the spectra of measured pressure are plotted in Figure 5. The amplitude of the pressure fluctuations at the bottom of the satellite mooring $\mathrm{I} 3$ $(253 \mathrm{~m})$ was about a factor of 2 less than the fluctuations at the bottom of the central mooring $(508 \mathrm{~m})$. This is expected if the moorings behaved as rigid pendulums. The same displacement spectra from Figure 4 are replotted in Figure 5 with no offset to show the small amount of contamination that is due to mooring motion. The contamination is even less for sensors near the top of the mooring where the swinging of the mooring is less than observed at the bottom.

All the spectra exhibit a change in slope near the local buoyancy frequency (Figure 4). In the internal wave frequency band from $f$ to $N$ the spectral slope is between -1 and -1.5 , significantly less steep than the -2 of the GM

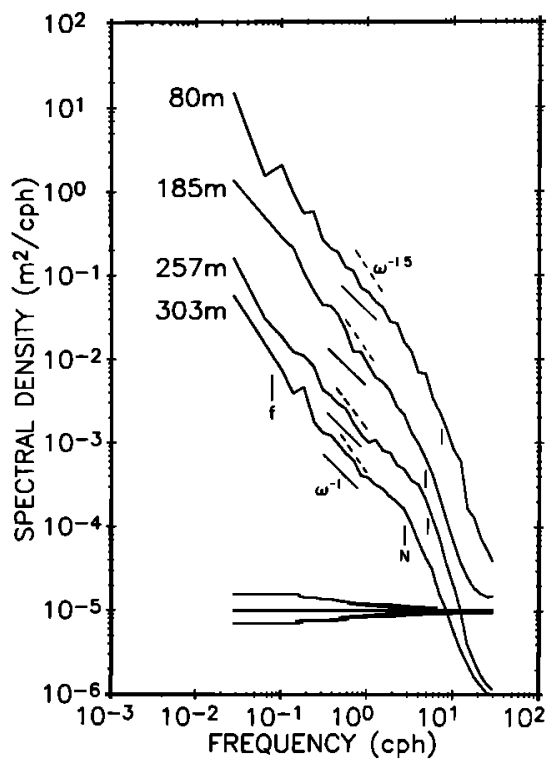

Fig. 4. Frequency spectra of vertical displacement inferred from time series of salinity measured on the central mooring at depths $80,185,257$, and $303 \mathrm{~m}$. The spectra at 185,257 , and $303 \mathrm{~m}$ are offset by factors of $10^{-1}, 10^{-2}$, and $10^{-3}$, respectively. Local values of buoyancy frequency $(N)$ are indicated. Spectral slopes of -1 and -1.5 are indicated. The range of the $95 \%$ confidence limits are given at the bottom.

model. Notably absent from the spectra are peaks at the $M_{2}$ tidal frequency and its harmonics; at lower latitude the internal tide is often a pronounced signal. It has even been suggested that the observed "continuous" internal wave spectrum is really composed of peaks at tidal harmonics that can be seen when suitably analyzed [Pinkel, 1983, 1984].

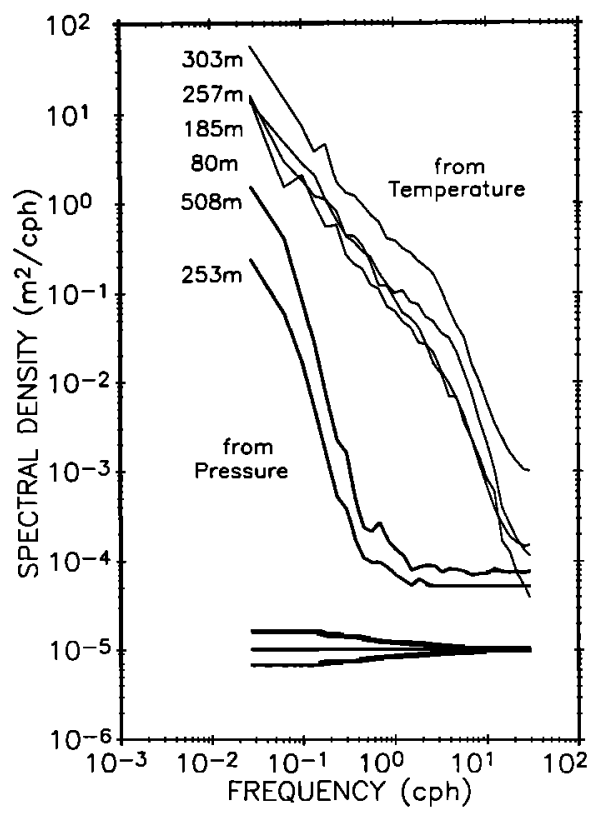

Fig. 5. Frequency spectra of pressure as measured by sensors at $253 \mathrm{~m}$ on satellite mooring 13 and at $508 \mathrm{~m}$ on the central mooring. Pressure is converted to depth using an average density typical of the region of $1028.13 \mathrm{~kg} \mathrm{~m}^{-3}$. For comparison, vertical displacement spectra shown in Figure 4 from the central mooring at 80, 185, 257, and $303 \mathrm{~m}$ are also plotted (no offset). 


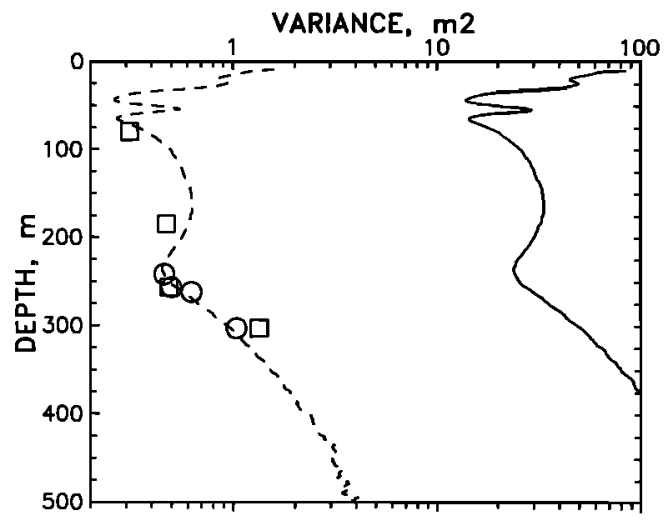

Fig. 6. Average vertical displacement variances estimated from time series of temperature (circles) and salinity (squares) are plotted with the variance of typical mid-latitude observations as characterized by the WKB-scaled Garrett-Munk model (solid line). Better agreement with the data is provided by the dashed line, which has the same shape as the solid line, but is reduced by a factor of 0.02 .

Note that at AIWEX the $M_{2}$ frequency is nearly the same as the inertial. Hence any tidal signal would have small vertical displacements and might not be resolved by these measurements anyway. The low amplitude of the $M_{2}$ internal tide may also be due to the weak barotropic tide in the Beaufort Sea [Kowalik and Untersteiner, 1978].

The vertical displacement variance in the internal wave band was estimated by integrating the frequency spectra of vertical displacement inferred from both salinity and temperature measurements where feasible (Figure 6). The depth dependence of the variance is proportional to $1 / N(z)$ as expected by the WKBJ approximation (equation 10). In order to compare with lower-latitude observations the variance predicted from the GM model

$$
\sigma_{G M}^{2}(z)=\frac{E b^{2} N_{0}}{2 N(z)}
$$

is also shown in Figure 6, using the canonical values of the parameters [Munk, 1981] $E=6.3 \times 10^{-5}$ (nondimensional energy), $b=1300 \mathrm{~m}$ (depth scale of $N(z)), N_{0}=5.23 \times 10^{-3}$ $\mathrm{s}^{-1}$ (3 cph) (buoyancy frequency scale). The observed variances are about a factor of 50 smaller than the GM level.

Vertical coherences were estimated between time series of vertical displacement measured in the central mooring. Only vertical separations of 5, 15 and 20 m between 242 and $262 \mathrm{~m}$ yielded nonzero coherences in the internal wave frequency band (Figure $7 b$ ). These coherences were estimated using the same data as used in the spectral estimates. The coherences show a strong peak near local $N$, which ranges from 5 to $7 \mathrm{cph}$. The same coherences are plotted as a function of vertical separation for various frequencies in Figure $7 a$. Below about $0.2 \mathrm{cph}$ the coherence cannot be estimated reliably even at a vertical separation of $5 \mathrm{~m}$. Fits of the data to the exponential form exp $[-a(\omega) \Delta z]$ are also shown.

Horizontal coherences were estimated between sensors on the satellite moorings. Because of the triangular array pattern each of the five horizontal separations were sampled at three orientations $120^{\circ}$ apart. For the most part there were no significant differences in the coherences or phase spectra for the different orientations at the same separation. Estimates of coherence for three horizontal separations are shown in Figure $8 b$; since there was not much variation, all three orientations at each separation were averaged together. In contrast to the vertical coherence, the horizontal coherences were higher at low frequency. The measured coherence was not significant above about $0.5 \mathrm{cph}$ at even the closest separation of $258 \mathrm{~m}$. At the largest separation of about $1 \mathrm{~km}$ the estimated coherence is not significant above $0.15 \mathrm{cph}$. The same coherence estimates plotted versus horizontal separation for various frequencies are shown in Figure $8 a$. An exponential form exp $[-b(\omega) r]$ is fit to all the data that were non-zero at the $95 \%$ significance level.

Are the observed vertical and horizontal coherences consistent with the behavior of random internal waves as hypothesized in section 2 ? To check the consistency, the energy distribution function $\bar{E}(\alpha, \omega)$ was estimated independently from vertical and horizontal coherences using (17). If consistent, estimates of $\bar{E}_{v}$ and $\bar{E}_{h}$ should be the same. However, since an exponential form was chosen for both horizontal and vertical coherences, $\bar{E}_{v}$ and $\bar{E}_{h}$ as defined in (17) cannot be equal for all $\alpha$ and $\omega$. Therefore it is techni-
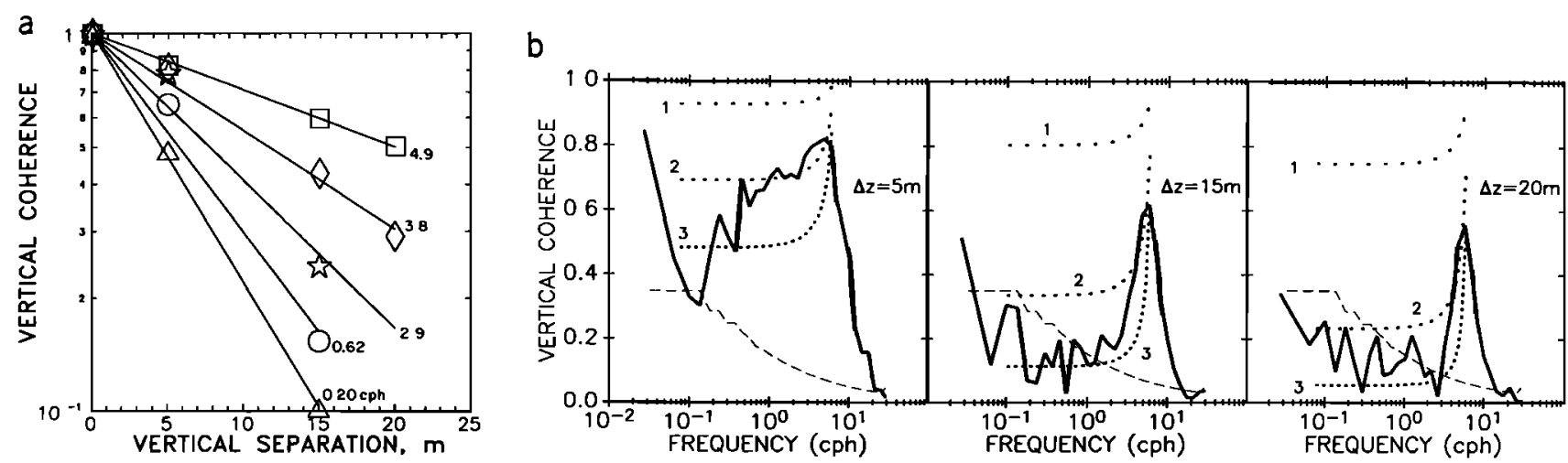

Fig. 7. Vertical coherences among vertical displacement series measured at 242,257 , and $262 \mathrm{~m}$ on the central mooring. (a) Coherence is plotted as a function of vertical separation at various frequencies. Exponential fits are also shown with straight solid lines. (b) Coherence is plotted as a function of frequency for three vertical separations. Data above the dashed line are significantly nonzero at the $90 \%$ confidence level. Model coherences with the same bandwidth (line 1), 5 times the bandwidth (line 2) and 10 times the bandwidth (line 3) of the GM79 model are also shown. 

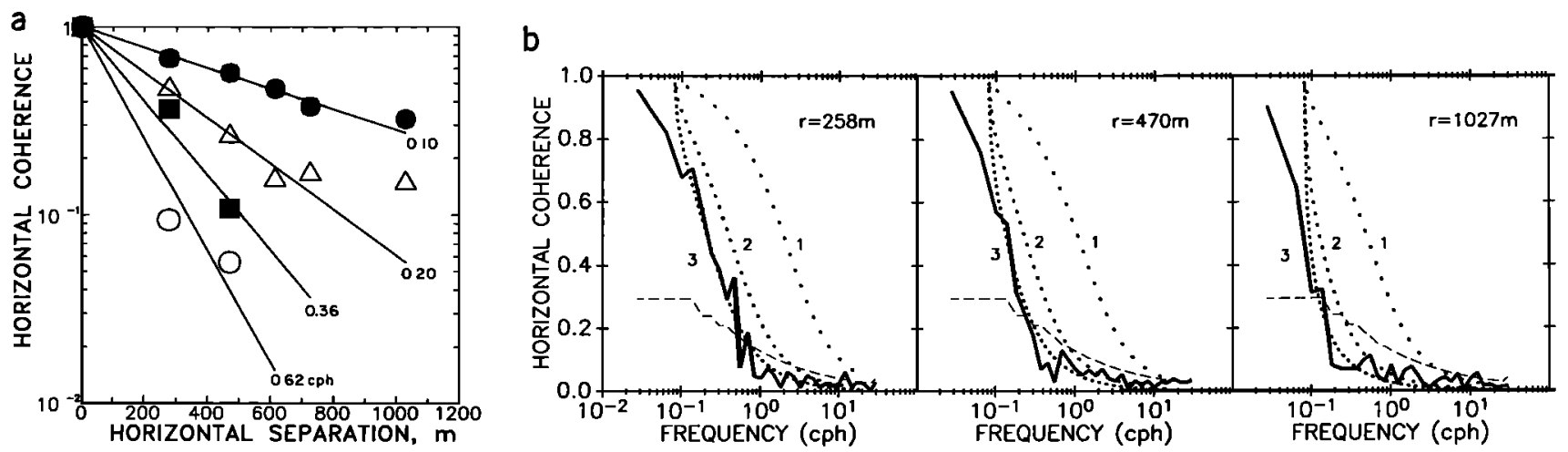

Fig. 8. Horizontal coherences among vertical displacement series measured at $249 \mathrm{~m}$ on the satellite moorings. (a) Coherence is plotted as a function of horizontal separation at various frequencies. Exponential fits are also shown with straight solid lines. (b) Coherence is plotted as a function of frequency for three horizontal separations. Data above the dashed line are significantly nonzero at the $90 \%$ confidence level. Model coherences with the same bandwidth (line 1 ), 5 times the bandwidth (line 2) and 10 times the bandwidth (line 3) of the GM79 model are also shown.

cally inconsistent to have forced both vertical and horizontal coherences to vary exponentially with separation.

To evaluate the ramifications of this inconsistency, consider the region of wavenumber space that is best resolved by the configuration of the sensors. The vertical coherences were only significantly resolved to $20 \mathrm{~m}$ separations; beyond $20 \mathrm{~m}$ the coherences were too small to estimate. Therefore, in evaluating the Fourier transform (equation (13)) the uncertainly of the coherences for $\Delta z>20 \mathrm{~m}$ most strongly affects the estimation of $\bar{E}_{v}(\alpha, \omega)$ for vertical wavelengths $>20 \mathrm{~m}$, or in terms of horizontal wavenumber, $\alpha<[\gamma(\omega) 20$ $\mathrm{m}]^{-1}$. Conversely, since there are no observations at vertical separations smaller than $5 \mathrm{~m}$, there is also an uncertainty in estimating $\bar{E}_{v}(\alpha, \omega)$ at large $\alpha$. Vertical wavelengths less than $5 \mathrm{~m}$, or equivalently, $\alpha>[\gamma(\omega) 5 \mathrm{~m}]^{-1}$ are not well-resolved. Hence the uncertainty in estimating $\bar{E}_{\nu}(\alpha, \omega)$ is greatest for $[\gamma(\omega) 5 \mathrm{~m}]^{-1}<\alpha<[\gamma(\omega) 20 \mathrm{~m}]^{-1}$.

A similar argument can be made to evaluate the uncertainty in estimating $\bar{E}_{h}$ from horizontal coherences. The uncertainty is large for horizontal wavelengths less than the minimum separation of about $250 \mathrm{~m}$, or $\alpha>[250 \mathrm{~m}]^{-1}$. Since the largest horizontal separation is about $1 \mathrm{~km}$, the uncertainty increases for $\alpha<[1000 \mathrm{~m}]^{-1}$. At high frequency the largest horizontal separation with significant coherence is less than $1 \mathrm{~km}$, which results in a larger range of uncertainty. Therefore estimates of $\bar{E}_{h}$, using the exponential form, become increasingly uncertain at $[250 \mathrm{~m}]^{-1}<\alpha<[1000$ $\mathrm{m}]^{-1}$.

Estimates of $\bar{E}_{v}(\alpha, \omega)$ and $\bar{E}_{h}(\alpha, \omega)$ calculated from (17) are plotted as a function of $\alpha$ for various frequencies in Figure $9 a, 9 b, 9 c$. Regions of $\bar{E}_{v}$ and $\bar{E}_{h}$ that are estimated with the most confidence are highlighted. $\bar{E}_{h}(\alpha, \omega)$ and $\tilde{E}_{v}(\alpha$, $\omega)$ compare favorably with each other at three different frequency bands. Hence, the inconsistency of forcing an exponential form for both horizontal and vertical coherences is not significant in the regions of $\alpha$ and $\omega$ that are best resolved by the data.

In order to compare these estimates of the energy distribution function $E(\alpha, \omega)$ with previous observations and models with different parameterizations, it is useful to define the equivalent statistical bandwidth $\alpha_{e}$ of the wave field [e.g., Blackman and Tukey, 1958] by

$$
\alpha_{e}(\omega)=\frac{\left[\int E(\alpha, \omega) d \alpha\right]^{2}}{\int E^{2}(\alpha, \omega) d \alpha}
$$

The quantity $\alpha_{e}$ represents the horizontal wavenumber bandwidth of an equivalent rectangular distribution function of $\alpha$ with the same ratio of variance to squared mean as $E(\alpha$, $\omega)$. This definition of bandwidth has been used previously to compare internal wave observations [e.g., Müller et al., 1978; Katz and Briscoe, 1979].

Using (19) and the analytical expressions for $\bar{E}_{v}(\alpha, \omega)$ and $\bar{E}_{h}(\alpha, \omega), \alpha_{e}$ can be related to the parameters of the exponential fit by

$$
\begin{aligned}
\alpha_{e_{v}} & =\frac{a(\omega)}{2 \gamma(\omega)} \\
\alpha_{e_{h}} & =\frac{8}{\pi^{2}} b(\omega)
\end{aligned}
$$

respectively (Figure 10a). Over the range of frequencies where estimates are made from both horizontal and vertical coherences, the agreement is good-within a factor of 2 . These results are also compared with estimates from other analyses (Figure 10a): GM75 [Garrett and Munk, 1975], GM79 [Munk, 1981; Desaubies, 1976], the Internal Wave Experiment (IWEX) [Briscoe, 1975; Müller et al., 1978], and the Mid-ocean Acoustic Transmission Experiment (MATE) [Levine et al., 1986b].

GM75 refers to the version of the GM model given in Garrett and Munk [1975]. The wavenumber dependence is given by

$$
\left(1+\frac{\alpha}{\alpha_{*}}\right)^{-5 / 2}
$$

where

$$
\alpha_{*}=\frac{\pi j_{*}}{N_{0} b}\left(\omega^{2}-f^{2}\right)^{1 / 2}
$$



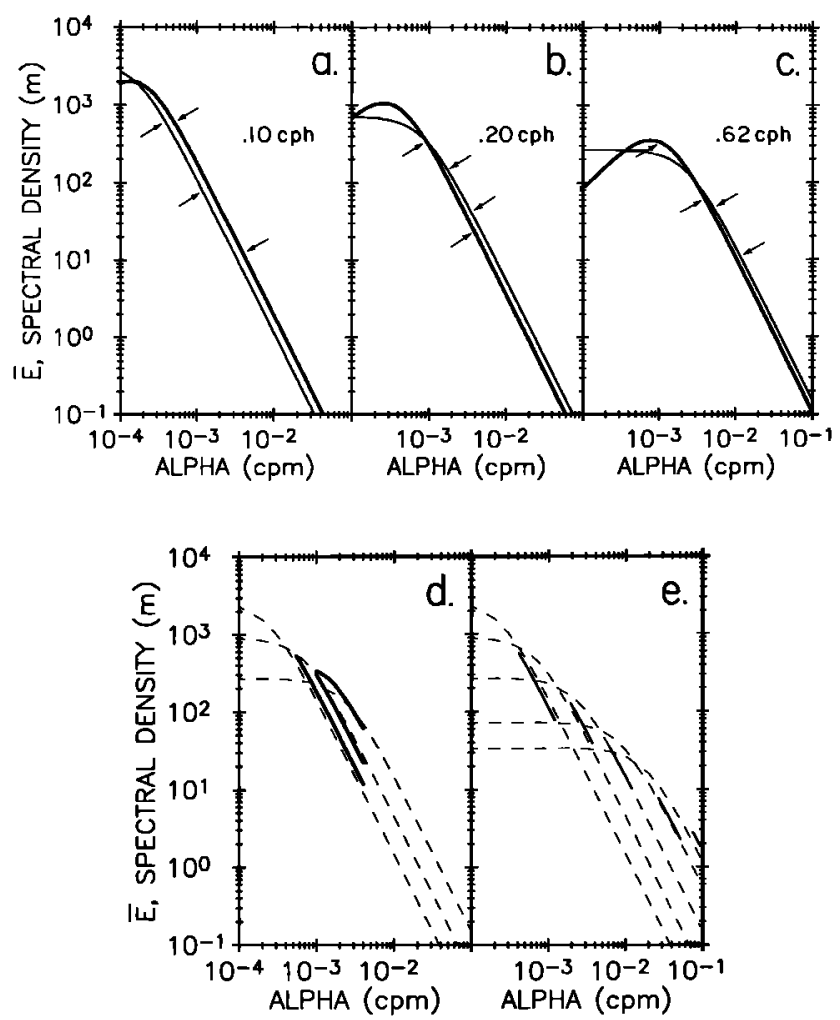

Fig. 9. Normalized spectral density estimated from horizontal coherences $\bar{E}_{h}$ (bold solid) and vertical coherences $\bar{E}_{v}$ (thin solid) is plotted as a function of horizontal wavenumber, $\alpha$, at frequencies $(a)$ $0.10 \mathrm{cph},(b) 0.20 \mathrm{cph}$, and (c) $0.62 \mathrm{cph}$. The region between the arrows is estimated with the most confidence. The normalized spectral density of the Garrett-Munk model (GM79) with 10 times the wavenumber bandwidth (dashed line) is compared with $(d) \bar{E}_{h}$ (bold solid) and $(e) \bar{E}_{u}$ (thin solid). Curves are plotted as a function of $\alpha$ for frequencies $(d) 0.10,0.20,0.62 \mathrm{cph}$, and $(e) 0.10,0.20,0.62$, 2.3 and $4.9 \mathrm{cph} . \bar{E}_{v}$ and $\bar{E}_{h}$ are shown only over the range of $\alpha$ that can be estimated with the most confidence.

is the horizontal wavenumber scale with the vertical mode scale $j_{*}=6$ providing the best fit to the data. This leads to

$$
\alpha_{e_{\mathrm{GM} 75}}=\frac{16}{9} \alpha_{*}
$$

For comparison in Figure 10a, the Arctic values of $N$ and $f$ are used; values of $N_{0} b$ and $j_{*}$ are the same as used in GM75.

An updated parameterization of the $\mathbf{G M}$ model, using discrete vertical modes, named GM79, was presented by Munk [1981]. The modifications were made to reflect recent observations, still mostly from mid-latitude. Desaubies [1976] proposed the same wavenumber dependence as GM79

$$
\left[1+\left(\frac{\alpha}{\alpha_{*}}\right)^{2}\right]^{-1}
$$

but still represented the vertical wavenumber as an equivalent continuous variable, thereby replacing the summation by a simpler integration. Evaluating $\alpha_{e}$ then yields

$$
\alpha_{e_{\mathrm{GM} 79}}=\pi \alpha_{*}
$$

where $j_{*}=3$ was chosen for best fit. In Figure $10 a$ the local
Arctic values of $N$ and $f$ are used with the GM79 parameters $N_{0} b$ and $j_{*}$.

Observations in the NE Pacific during MATE indicated a good fit was obtained using the same wavenumber dependence as GM79 but with $j_{*}=6$, indicating twice the horizontal wavenumber bandwidth given by GM79.

The data from the Sargasso Sea during IWEX was modeled with a more complicated wavenumber dependence

$$
\left\{1+\left(\frac{\alpha-\alpha_{p}}{\alpha_{*}}\right)^{s}\right\}^{-t / s} \alpha>\alpha_{p}
$$

where $\alpha_{p}$ is a low wavenumber cutoff. The parameters $\alpha_{p}, t$, and $s$ were fit at each frequency; Müller et al. [1978] estimate the bandwidth to be

$$
\alpha_{e_{\text {IWEX }}}=\frac{j_{e} \pi}{\left(N_{0} b\right)_{\text {IWEX }}}\left(\omega^{2}-f^{2}\right)^{1 / 2}
$$

where

$j_{e}=j_{\infty}+\frac{1}{2}\left(j_{0}+j_{\infty}\right)\left[1-\tanh \frac{\log \omega / f-\log \omega_{m} / f}{\frac{1}{4} \log \omega_{\infty} / \omega_{0}}\right]$

with $j_{\infty}=10, j_{0}=20, \omega_{m}=0.173 \mathrm{cph}, \omega_{\infty}=1.133 \mathrm{cph}$, $\omega_{0}=0.44 \mathrm{cph}$ and $\left(N_{0} b\right)_{\text {IWEX }}=875 \mathrm{~m} \mathrm{~h}^{-1}$. Unlike the GM model the mode scale $j_{e}$ is a function of frequency with fewer modes at high frequency. It is more difficult to scale this energy distribution to the Arctic values of $N$ and $f$ since the dependence of the parameters $j_{0}, j_{\infty}, \omega_{m}, \omega_{\infty}$, and $\omega_{0}$ on $N$ and $f$ is not known. Therefore, the same values of these parameters given by Müller et al. [1978] are used in Figure $10 a$; the Arctic values of $N$ and $f$ are used to calculate $\alpha_{e_{\text {IWEX }}}$.

Clearly, the horizontal wavenumber bandwidth measured during AIWEX is significantly greater than any estimates from lower latitudes. The AIWEX bandwidth is about a factor of 10 greater than the GM79 or GM75 models. The same information can also be expressed in terms of equivalent vertical bandwidth using the WKBJ approximation (Figure 10b).

The increased bandwidth indicates that there is a wider distribution of wavelengths present at each frequency; this creates a more confused sea of waves and leads to lower coherences. The differences between the AIWEX results and the GM models is dramatically demonstrated by plotting the GM model coherences with the AIWEX observations. Using the parameter values of GM79, it is clear that the GM model would predict much greater vertical and horizontal coherences than were observed (Figures $7 b$ and $8 b$ ). However, if the bandwidth is increased by a factor of 10 (equivalent in GM79 to increasing $j_{*} / N_{0} b$ by a factor of 10 ), the GM model agrees favorably with the AIWEX coherence structure. A comparison of $\bar{E}_{v}$ and $\bar{E}_{h}$ estimated from AIWEX with GM79 with the increased bandwidth is made in Figures $9 d$ and $9 e$. The agreement is good over the most reliable range of the estimates of $\bar{E}_{v}$ and $\bar{E}_{h}$.

A detailed error analysis of the fitting procedure used to estimate $\tilde{E}_{v}$ and $\bar{E}_{h}$ was not practical. It is clear by comparing the model coherences from GM79 that there are large and significant departures in the AIWEX data (Figures 6 and 7). An interesting question is how much of the wave field is consistent with internal wave kinematics? The answer, un- 
fortunately, is not easily obtained. The data are sufficiently sparse that all the observations can be explained as internal waves. More data, such as horizontal velocity, would constrain the interpretation and perhaps lead to an estimate of the non-internal wave signal.

One well-known feature of the GM parameterization is that the energy distribution function $E(\beta, \omega)$ is separable, that is, it can be written as a product $E(\beta, \omega)=g(\beta) h(\omega)$. It then follows that the vertical coherence is a function of vertical separation only independent of frequency. Also, the vertical wavenumber bandwidth is independent of frequency. Therefore the observations (Figures 7 and 10) indicate that $E(\beta, \omega)$ is not completely separable, especially at frequencies near $N$. It is not unexpected that the model is not valid near $N$ as the hydrostatic approximation $\left(N^{2}-\right.$ $\left.\omega^{2}\right)^{1 / 2} \sim N$ has been used in the derivation. However, if one relaxes this assumption, as was done by Desaubies [1976], then the GM model exhibits some frequency dependence in vertical coherence and vertical wavenumber bandwidth. The model results displayed in Figure 10, with a relaxed hydrostatic assumption, mimic the frequency dependence of the observation near $N$. The decrease in vertical wavenumber bandwidth at high frequency appears to be a universal feature [e.g., Pinkel, 1975; Käse and Siedler, 1980; Levine et al., $1983 b]$.

A further test of the efficacy of the fundamental relationship between the energy distribution $E(\alpha, \omega)$ and the coherence $C(r, \Delta z, \omega)$ is provided by examining slant coherences. Slant coherence is defined to be the coherence between two locations separated both vertically and horizontally. Examples of observed slant coherences with $\Delta z=8 \mathrm{~m}$ and $r=595$ $\mathrm{m}$ and with $\Delta z=4 \mathrm{~m}$ and $r=150 \mathrm{~m}$ are shown in Figure 11 . The GM79 prediction with increased bandwidth agrees remarkably well with the observations. Even though the vertical separation is relatively small, it is clearly different from a pure horizontal coherence with no vertical separation, especially at low frequency.

An independent estimate of wavenumber bandwidth was made during AIWEX by M. D. Morehead and E. A. D'Asaro (Vertical and horizontal scales of internal waves at AIWEX, submitted to Journal of Geophysical Research, 1989). Using vertical wavenumber spectra of horizontal kinetic energy from Expendable Current Profilers (XCP), they estimate the bandwidth to be between 3 and 10 times greater than the GM75 model. These estimates are consistent with the analysis of the moored observations presented here.

The goal of this analysis was to determine the frequencywavenumber composition of the internal wave field based on measurements of vertical and horizontal coherences and the theoretical assumptions stated in section 3 . Given the limited number of horizontal and vertical separations, the determination of $\bar{E}(\alpha, \omega)$ is correspondingly coarse. If more data were available, then a more precise determination of $\bar{E}$ would be possible. Few direct estimates of the joint frequency-wavenumber distribution of the wave field have been made, most notably by Pinkel [1983, 1984, 1985]. As might be anticipated, the higher the resolution of the data, the more complicated the result. Pinkel [1984] suggests that classifying the frequency-wavenumber distribution by an equivalent wavenumber bandwidth may be misleading. Clearly, significantly diverse wavenumber distributions can have the same equivalent bandwidth. In this analysis the imposed exponential form of the coherence structure at all frequencies has
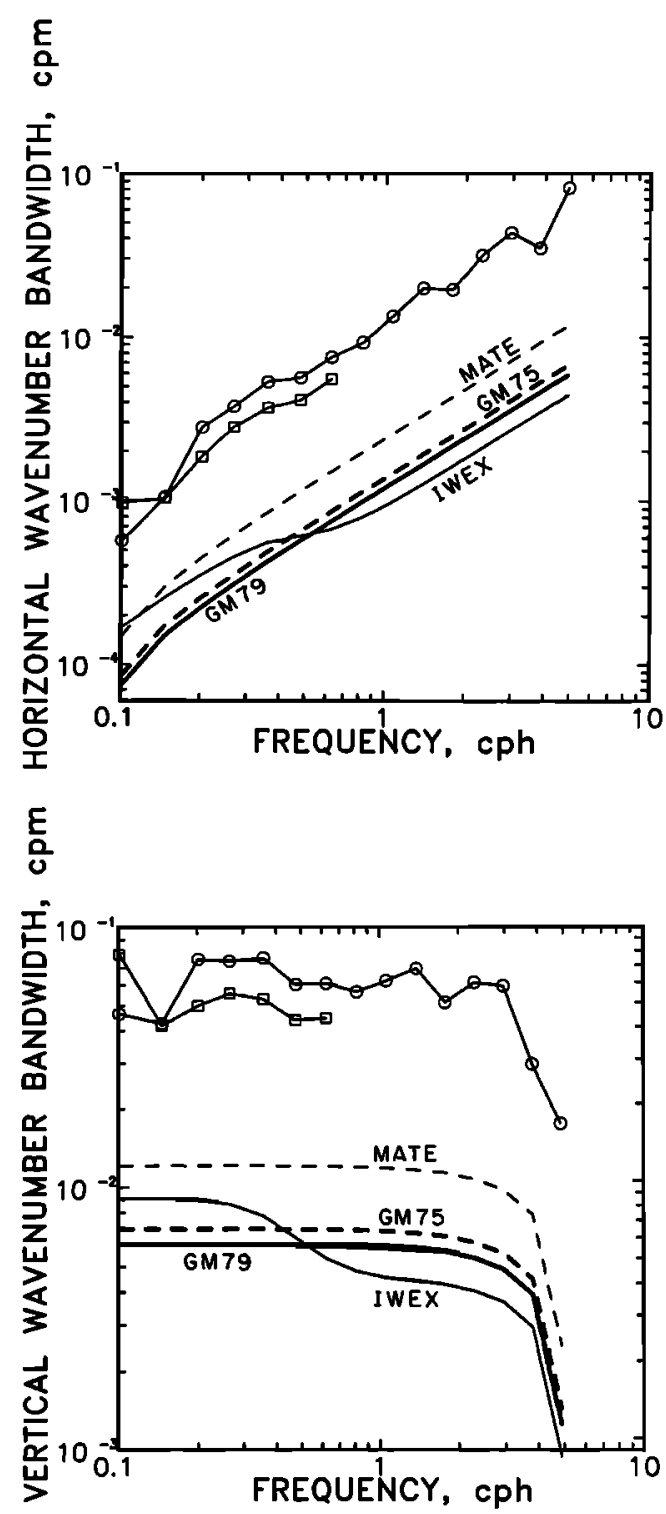

Fig. 10. Equivalent bandwidth of (a) horizontal wavenumber and $(b)$ vertical wavenumber as estimated from vertical coherence data (circles) and horizontal coherence data (squares). Comparison is made with bandwidths from other models and experiments: IWEX [Müller et al., 1978]; GM75 [Garrett and Munk, 1975]; GM79 [Munk, 1981]; MATE [Levine et al., 1986b].

forced $\bar{E}(\alpha, \omega)$ to have nearly the same functional dependence on $\alpha$. However, the $\omega$ dependence of $\bar{E}$ is determined from the frequency dependence of the data to the exponential fit, namely $a(\omega)$ and $b(\omega)$. Although calculating $\bar{E}$ by directly transforming the data (equations (13) and (15)) is not possible, the cruder estimates made using the exponential fits indicate that the bandwidth is markedly wider at AIWEX compared to lower latitudes.

For future comparisons it is useful to express explicitly the energy distribution $E(\alpha, \omega)$ or $E(\beta, \omega)$. To accomplish this, the frequency spectrum in Figure 4 is described by the function

$$
\frac{S(0, z, z, \omega)}{\nu^{2}(z)}=A \frac{\left(\omega^{2}-f^{2}\right)^{1 / 2}}{\omega^{2.2}}
$$




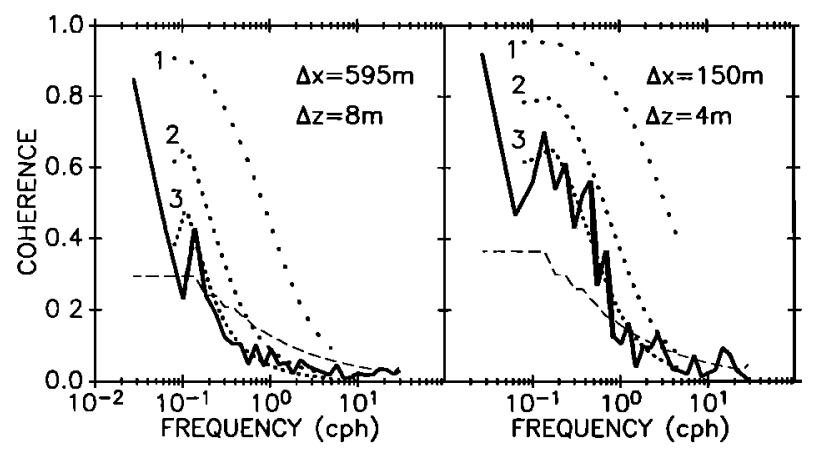

Fig. 11. Slant coherences of vertical displacement as a function of frequency. (a) Coherence is plotted between series at $257 \mathrm{~m}$ on the central mooring and at $249 \mathrm{~m}$ on the outer satellite moorings at a horizontal separation of $595 \mathrm{~m}$. (b) Coherence is plotted between series at $257 \mathrm{~m}$ on the central mooring and at $253 \mathrm{~m}$ on the inner satellite mooring 13. Data above the dashed line are significantly nonzero at the $90 \%$ confidence level. Model coherences with the same bandwidth (line 1), 5 times the bandwidth (line 2) and 10 times the bandwidth (line 3) of the GM79 model are also shown.

where $A=3.3 \times 10^{-2} \mathrm{~m}^{2} \mathrm{~s}^{-.2}$ and the buoyancy frequency at $257 \mathrm{~m}$ is taken as the reference, i.e., $N(257 \mathrm{~m})=\bar{N}=10^{-2}$ $\mathrm{s}^{-1}(5.7 \mathrm{cph})$. At $\omega \gg f$ the spectral slope approaches -1.2 . An "inertial cusp", i.e., $\left(\omega^{2}-f^{2}\right)^{1 / 2}$, is included primarily to facilitate comparison with the GM formulation even though its existence is not confirmed by the data itself.

The energy distribution of vertical displacement then becomes (equations (13), (15))

$$
E(\alpha, \omega)=\bar{E}(\alpha, \omega) A \frac{\left(\omega^{2}-f^{2}\right)^{1 / 2}}{\omega^{2.2}}
$$

or

$$
E(\beta, \omega)=\bar{E}(\beta, \omega) A \frac{\left(\omega^{2}-f^{2}\right)^{1 / 2}}{\omega^{2.2}}
$$

where either $\bar{E}_{h}$ or $\bar{E}_{v}$ can be used for $\bar{E}$ (equation (17)).

\section{Summary and Conclusions}

The spectral composition of the internal wave field under the Arctic peak ice during AIWEX was found to be strikingly different from observations at lower latitudes. Time series of vertical displacement were inferred from horizontal and vertical arrays of temperature and conductivity sensors. Estimates of the average frequency autospectra of vertical displacement revealed that (1) the frequency spectrum has a spectral slope near -1.2 (Figure 4); whiter than at lower latitudes, (2) the total displacement is a factor of 0.02 lower than the GM model, yet still scales with depth as the WKBJ approximation predicts (Figure 6), and (3) there are no peaks at the $M_{2}$ tidal frequency and its harmonics.

An analysis of the coherences between the vertically and horizontally separated sensors showed that (1) the wave field is horizontally isotropic, based on estimates from three orientations in the horizontal, (2) the coherence structure is consistent with the assumptions of a random, linear internal wave field, and (3) the observed low coherence implies that the wavenumber bandwidth is about a factor of 10 higher than at lower latitude (Figures 7, 8, 9, and 10).

Why is the nature of the internal wave field measured during AIWEX so different from lower latitude observations? There are many possibilities that may be responsible for reduced internal wave variance, as suggested by Levine et al. [1987], including (1) the presence of the ice cover increasing dissipation over that of an ice-free ocean [Morison et al., 1985], (2) the reduced amount of stress that is transferred to the water because the winds are weaker [Paulson, 1980], and some momentum is transmitted to the coast, (3) the small barotropic tide [Kowalik and Untersteiner, 1978] which leads to weak internal tides, and (4) the weak, large-scale circulation of the Arctic Ocean compared to most oceans [Coachman and Aagaard, 1974].

Whatever the reason, it appears that the forcing at AIWEX is reduced from the forcing at lower latitude. Perhaps the wave field is undersaturated and has a spectral shape (slope near -1 ) that is characteristic of the forcing mechanism. One might speculate that if forcing is increased, the wave field would approach the GM level and spectral slope (saturation).

These results invite examination by theoreticians studying nonlinear interactions. The observations at AIWEX describe an internal wave field that differs significantly from the canonical GM, mid-latitude model. The implications of the existence of this unique wave field on the theoretical understanding of internal waves are of interest.

This study focused on the average statistical quantities. Averages are useful when comparing with other similar analyses of internal waves. The surprising results put forth by GM was that the internal wave field is remarkably constant in time and space; the internal wave field in any ocean at any time seemed to nearly follow the features of the GM formulation. To obtain clues toward understanding the mechanisms that generate internal waves, it is perhaps more enlightening to examine the time dependence of the frequency-wavenumber composition. Indeed, the wave field did vary significantly in time during AIWEX. The amplitude of the waves appears to be correlated with the moving ice, suggesting generation from under-ice topography. Such a study is being actively pursued.

Acknowledgments. I thank James Morison for providing enthusiasm and guidance during the planning of AIWEX and for being an "Arctic hero" role-model throughout the experiment. Special thanks are extended to Clayton Paulson, Jay Simpkins, Steve Gard, and Rick Baumann without whose expertise and muscle this project would not have succeeded. The excellent administration of the logistical support by Andy Heiberg. Allen Hielscher, and Imants Virsnieks made the AIWEX camp a comfortable place to be, even though "it's no picnic out there." The endless toil of Matt Valley in running the mess hall and providing a philosophical perspective is appreciated. Support for this project by the Office of Naval Research through contracts N00014-84-C-0218 and N00014-87-K-0009 is gratefully acknowledged.

\section{REFERENCES}

Blackman, R. B., and J. W. Tukey, The Measurement of Power Spectra from the Point of View of Communication Engineering, Dover, New York, 1958.

Bracewell, R. N., The Fourier Transform and Its Application, 444 pp., McGraw-Hill, New York, 1978.

Briscoe, M. G., Preliminary results from the tri-moored Internal Wave Experiment (IWEX), J. Geophys. Res., 80, 3872-3884, 1975.

Coachman, L. K., and K. Aagaard, Physical oceanography of Arctic and subarctic seas, in Marine Geology and Oceanography 
of the Arctic Seas, edited by Y. Herman, pp. 1-72, SpringerVerlag, New York, 1974.

Desaubies, Y. J. F., Analytical representation of internal wave spectra, J. Phys. Oceanogr., 6, 976-981, 1976.

Garrett, C. J. R., and W. H. Munk, Space-time scales of internal waves, Geophys. Astrophys. Fluid Dyn., 2, 255-264, 1972.

Garrett, C. J. R., and W. H. Munk, Space-time scales of internal waves: A progress report, J. Geophys. Res., 80, 291-297, 1975.

Gill, A. E., Atmosphere-Ocean Dynamics, 662 pp., Academic, San Diego, Calif., 1982.

Gradshteyn, I. S., and I. M. Ryzhik, Table of Integrals, Series, and Products, 1086 pp., Academic, San Diego, Calif., 1965.

Käse, R. H., and G. Siedler, Internal wave kinematics in the upper tropical Atlantic, Deep Sea Res., 26, GATE suppl. I, 161-189, 1980.

Katz, E. J., and M. G. Briscoe, Vertical coherence of the internal wave field from towed sensors, J. Phys. Oceanogr., 9, 518-530, 1979.

Kowalik, Z., and N. Untersteiner, A study of the $M_{2}$ tide in the Arctic Ocean, Dtsch. Hydrogr. Z., 31, 216-229, 1978.

Levine, M. D., R. A. deSzoeke, and P. P. Niiler, Internal waves in the upper ocean during MILE, J. Phys. Oceanogr., 13, 240-257, $1983 a$.

Levine, M. D., C. A. Paulson, M. G. Briscoe, R. A. Weller, and H. Peters, Internal waves in JASIN, Philos. Trans. R. Soc. London, Ser. A, 308, 389-405, $1983 b$.

Levine, M. D., C. A. Paulson, and J. H. Morison, Internal waves in the Arctic Ocean: Comparison with lower-latitude observations, J. Phys. Oceanogr., 15, 800-809, 1985.

Levine, M. D., S. R. Gard, and J. Simpkins, Moored temperature and conductivity observations during AIWEX Data Rep. 123, Ref. 86-9, 195 pp., Coll. of Oceanogr., Oregon State Univ., Corvallis, 1986a.

Levine, M. D., J. D. Irish, T. E. Ewart, and S. A. Reynolds, Simultaneous spatial and temporal measurements of the internal wave field during MATE, J. Geophys. Res., 91, 9709-9719, 1986 b.

Levine, M. D., C. A. Paulson, and J. H. Morison, Observations of internal gravity waves under the Arctic pack ice, J. Geophys. Res., 92, 779-782, 1987.

Morison, J. H., C. E. Long, and M. D. Levine, Internal wave dissipation under sea ice, J. Geophys. Res., 90, 11,959-11,966, 1985.

Müller, P., D. J. Olbers, and J. Willebrand, The IWEX spectrum, $J$. Geophys. Res., 83, 479-500, 1978.

Müller, P., G. Holloway, F. Henyey, and N. Pomphrey, Nonlinear interactions among internal gravity waves, Rev. Geophys., 24, 493-536, 1986.

Munk, W. H., Internal waves and small-scale processes, in Evolution of Physical Oceanography, edited by B. A. Warren and C. Wunsch, pp. 264-290, MIT Press, Cambridge, Mass., 1981.

Munk, W., and N. Phillips, Coherence and band structure of inertial motion in the sea, Rev. Geophys., 6, 447-472, 1968.

Paulson, C. A., A review of the AIDJEX atmospheric program, in Sea Ice Processes and Models, edited by R. S. Pritchard, pp. 28-33, University of Washington Press, Seattle, 1980.

Pinkel, R., Upper ocean internal wave observations from FLIP, $J$. Geophys. Res., 80, 3892-3910, 1975.

Pinkel, R., Doppler sonar observations of internal waves: Wavefield structure, J. Phys. Oceanogr., 13, 804-815, 1983.

Pinkel, R., Doppler sonar observations of internal waves: The wavenumber-frequency spectrum, J. Phys. Oceanogr., 14, 1249$1270,1984$.

Pinkel, R., A wavenumber-frequency spectrum of upper ocean shear, J. Phys. Oceanogr., 15, 1453-1469, 1985.

Wunsch, C., Deep ocean internal waves: What do we really know?, J. Geophys. Res., 80, 339-343, 1975.

Wunsch, C., Geographical variability of the internal wave field: A search for sources and sinks, J. Phys. Oceanogr., 6, 471-485, 1976.

Wunsch, C., and S. Webb, The climatology of deep ocean internal waves, J. Phys. Oceanogr., 9, 235-243, 1979.

M. D. Levine, Oceanography Administration Building 104, College of Oceanography, Oregon State University, Corvallis, OR 97331 .

(Received June 26, 1989; revised November 2, 1989; accepted August 23, 1989.) 